\title{
Numerical Solution of Falkner-Skan Equation by Iterative Transformation Method
}

\section{Helmi Temimi and Mohamed Ben-Romdhane}

International Centre for Applied Mathematics and Computational Bioengineering, Department of Mathematics and Natural Sciences, Gulf University for Science and Technology

P.O. Box 7207, 32093 Hawally, Kuwait

E-mail(corresp.): temimi.h@gust.edu.kw

E-mail: romdhane.m@gust.edu.kw

Received June 19, 2017; revised December 5, 2017; accepted December 7, 2017

\begin{abstract}
In this paper, we study the nonlinear boundary-layer equation of FalknerSkan defined on a semi-infinite domain. An iterative finite difference (IFD) scheme is proposed to numerically solve such nonlinear ordinary differential equation. A computational iterative scheme is developed based on Newton-Kantorovich quasilinearization. At every iteration, the obtained linearized differential equation is numerically solved using the standard finite difference method. Numerical experiments show the accuracy and efficiency of the method compared to existing solvers. The computation is performed for different parameter values, including the special case of Blasius problem.
\end{abstract}

Keywords: Falkner-Skan equation, Blasius equation, quasi-linearization, iterative finite difference method.

AMS Subject Classification: 34B15; 65D15; 65L10; 65L12.

\section{Introduction}

The numerical solution for nonlinear differential equations is a major problem in computational mathematics. Specifically, nonlinear boundary value problems on infinite domain attract a major attention of the scientific community. One known nonlinear differential equation defined on a semi-infinite domain is the so-called Blasius equation which appeared in the literature in 1908 [4]. Blasius equation describes the viscous flow in a laminar boundary layer over a flat plate. Because of its interesting application to fluid flow, physicists as well as engineers have been always keenly interested in solving this nonlinear ordinary

Copyright (c) 2018 The Author(s). Published by VGTU Press

This is an Open Access article distributed under the terms of the Creative Commons Attribution License (http://creativecommons.org/licenses/by/4.0/), which permits unrestricted use, distribution, and reproduction in any medium, provided the original author and source are credited. 
differential equation rich in physical, mathematical, and numerical challenges. Blasius equation is part of the set of more general nonlinear problems known as the Falkner-Skan equations [7]. The third-order nonlinear equation of FalknerSkan type is a famous example of nonlinear boundary value problems on semiinfinite domains arising from the boundary layer flow with stream-wise pressure gradient. It is governed by the following equation:

$$
\frac{d^{3} f}{d \eta^{3}}+\beta_{0} f \frac{d^{2} f}{d \eta^{2}}+\beta_{1}\left[1-\left(\frac{d f}{d \eta}\right)^{2}\right]=0, \quad 0<\eta<\infty
$$

subject to the boundary conditions

$$
f(0)=0, \quad f^{\prime}(0)=\gamma, \quad \lim _{\eta \rightarrow+\infty} f^{\prime}(\eta)=1,
$$

where $\gamma$ is the movement velocity ratio of the plate to the mainstream.

In fact, when $0<\gamma<1$, the speed of oncoming fluid is larger than the speed of the plate; however, when $\gamma>1$, the speed of the moving plate is faster than the speed of the oncoming fluid. Furthermore, the case when $\gamma<0$ corresponds to a movement of the plate and the mainstream in opposite directions. Particularly, the special case $\gamma=0$ (for a fixed plate) and $\beta_{1}=0$ leads to Blasius problem [4].

Due to its challenging physical properties, many researchers have attempted to solve Falkner-Skan equation with various methods. Among these attempts, we can cite the work of Hartree [11] who showed that under some conditions there exists a family of unique solutions of the problem whose first-order derivative tends to 1 exponentially. Howarth [14] attempted also to solve the boundary layer problem in a retarded region by reducing the problem to the solution of a first order differential equation.

Later, Falkner-Skan problem has attracted the attention of more researchers such as Cebeci and Keller [5], Asaithambi [2], and Sher and Yakhot [27] who applied various numerical approaches to solve this problem. Moreover, Liao $[15,16]$ applied the homotopy analysis method to solve the Falkner-Skan equation which provided an explicit analytical solution with the same boundary conditions as those of above. Additionally, Salama [26] proposed a higher-order method for solving such free boundary problems. Sachdev et al [25] proposed also an exact solution to Falkner-Skan equation subject to appropriate physical boundary conditions arising from boundary layer theory. Besides, Liu and Chang [17] investigated numerically the solutions of the Falkner-Skan equation with the boundary conditions of resting and impermeable wall. Moreover, Zhu et al. [30] presented two iterative methods for solving the Falkner-Skan equation based on the quasilinearization technique and a cubic spline solver. The original problem has been formulated as a new free boundary value problem. Additionally, an adaptive algorithm for the Thomas-Fermi equation by means of the moving mesh finite element method has been developed [31].

Recently, Fang et al. [8,9] have studied analytically a special case of the Falkner-Skan equation using $\beta_{1}=-1$ with the boundary conditions of mass transfer and wall stretching, as well as the problem of a shrinking sheet with a constant sheet velocity. On the other hand, different numerical methods have been applied in order to find accurate solutions, such as Runge-Kutta 
algorithm [6], recursive evaluation of Taylor coefficients [1], and iterative transformation method [10]. Furthermore, other types of similar flows has been recently treated such as the work of Raju et al. who studied the nonlinear flows of Casson and Carreau fluids on various types of wedges [20,21,22,23,24]. Also, Mukhopadhyay et al. [18] investigated a boundary-layer forced convection flow of a Casson fluid past a symmetric wedge.

Despite these aforementioned research work since the beginning of the previous century, there is no closed-form solutions available for Falkner-Skan problem nor for the special case of Blasius problem. This fact stimulated researchers to investigate for most reliable, efficient, and low-computation cost solutions. In this manuscript, we introduce a new coordinate transformation and we apply it to Falkner-Skan problem (1.1) in order to overcome the difficulty of the semi-infinite domain. Using the aforementioned transformation and the introduced auxiliary function, a system of nonlinear differential equations is obtained leading to a second order nonlinear integro-differential equation subjected to boundary conditions defined on a finite values 0 and 1 . In order to solve the resulting nonlinear integro-differential equation, we develop an iterative scheme based on Newton-Raphson-Kantorovich method in function space [3,28] combined with the standard finite difference method. Numerical simulations will be performed to demonstrate the efficiency and reliability of the proposed numerical solver in producing accurate solutions with low computation cost.

\section{Transformed problem and iterative finite difference solution}

\subsection{Order reduction and coordinate transformation}

In order to perform order reduction of the problem (1.1), we introduce an auxiliary function $g$ defined by $g(\eta)=f^{\prime}(\eta)$ to obtain the following nonlinear system

$$
\left\{\begin{array}{l}
\frac{d^{2} g}{d \eta^{2}}+\beta_{0} f \frac{d g}{d \eta}+\beta_{1}\left(1-g^{2}\right)=0 \\
f^{\prime}-g=0
\end{array}\right.
$$

subject to the boundary conditions

$$
f(0)=0, \quad g(0)=\gamma, \quad \lim _{\eta \rightarrow+\infty} g(\eta)=1 .
$$

The solution of Falkner-Skan problem is sought on a semi-infinite domain $\eta \geq 0$. Moreover, one of the boundary conditions is asymptotically assigned on the first derivative at infinity. These two aforementioned characteristics of the problem are the main challenges to be faced on handling this type of problems. Therefore, we define a coordinate transformation by introducing a new variable $t$ in order to map the changing physical domain $[0, \infty)$ to a fixed computational domain $[0,1)$. This new variable is defined as

$$
t=\eta /(\eta+1)
$$


It is clear that the mapping $t:[0, \infty) \longrightarrow[0,1), \eta \longrightarrow \eta /(\eta+1)$ is one-to-one. Then, given $t, \eta$ is obtained as $\eta=t /(1-t)$. We also introduce two functions $h(t)$ and $k(t)$ defined by

$$
\begin{aligned}
& f(\eta)=f(\eta(t))=f(t /(1-t))=h(t), \\
& g(\eta)=g(\eta(t))=g(t /(1-t))=k(t) .
\end{aligned}
$$

As a result, we have

$$
\begin{aligned}
f^{\prime}(\eta) & =\frac{d f}{d t} \frac{d t}{d \eta}=h^{\prime}(t) \frac{d t}{d \eta} \\
& =\frac{1}{(\eta+1)^{2}} h^{\prime}(t)=(1-t)^{2} h^{\prime}(t) .
\end{aligned}
$$

Similarly,

$$
\begin{aligned}
g^{\prime}(\eta) & =(1-t)^{2} k^{\prime}(t), \\
g^{\prime \prime}(\eta) & =\left[(1-t)^{2} k^{\prime \prime}(t)-2(1-t) k^{\prime}(t)\right](1-t)^{2} \\
& =(1-t)^{4} k^{\prime \prime}(t)-2(1-t)^{3} k^{\prime}(t) .
\end{aligned}
$$

Hence, using (2.2) and (2.3) in the system of nonlinear differential equations (2.1) leads to the following

$$
\left\{\begin{array}{l}
(1-t)^{4} k^{\prime \prime}(t)-2(1-t)^{3} k^{\prime}(t)+\beta_{0} h(t) k^{\prime}(t)-\beta_{1} k^{2}(t)=-\beta_{1}, \\
(1-t)^{2} h^{\prime}(t)-k(t)=0
\end{array}\right.
$$

subject to the boundary conditions

$$
h(0)=0, \quad k(0)=\gamma, \quad k(1)=1 .
$$

Using the boundary condition $h(0)=0$, the second equation in the system (2.4a) becomes

$$
h(t)=\int_{0}^{t} \frac{1}{(1-\epsilon)^{2}} k(\epsilon) d \epsilon .
$$

Thus, by combining both equations of the system (2.4a), the problem (2.4) can be written in the form of a second order nonlinear integro-differential equation defined for $t \in(0,1)$ as

$$
\begin{aligned}
(1-t)^{4} k^{\prime \prime}(t)-2(1-t)^{3} k^{\prime}(t) & -\beta_{1} k^{2}(t)+\beta_{1} \\
& +\beta_{0}(1-t)^{2} k^{\prime}(t) \int_{0}^{t} \frac{k(\epsilon)}{(1-\epsilon)^{2}} d \epsilon=0
\end{aligned}
$$

and subject to the boundary conditions

$$
k(0)=\gamma, \quad k(1)=1 .
$$

In summary, we reduced the Falkner-Skan problem (1.1) of third order, defined on a semi-infinite domain with an asymptotic boundary condition, to a second 
order nonlinear integro-differential equation (2.6) defined over a finite domain and subjected to two boundary conditions given at finite values 0 and 1 . To determine the solution of problem (1.1), we have to solve problem (2.6) for $k(t)$, recuperate $h(t)$ using $(2.5)$, then compute $f(\eta)$ using the relation

$$
f(\eta)=h(t)=h(\eta /(\eta+1)), \quad \forall \eta \in(0,+\infty)
$$

\subsection{Quasi-linearization of the transformed Falkner-Skan problem}

In this section, we apply the Newton-Raphson-Kantorovich approximation in function space introduced by $[3,28]$ on the newly introduced second order integro-differential equation (2.6) resulting in the previous section. At every iteration, the obtained quasi-linearized differential equation is numerically solved using the finite difference method yielding the solution of the transformed Falkner-Skan's boundary value problem (2.1).

First, let us recall the Newton-Raphson-Kantorovich approximation method in function space to solve nonlinear differential equations. Let $\left\{v_{n}\right\}$ be a sequence of approximate solutions of the nonlinear ordinary differential equation $F\left(v, v^{\prime}, v^{\prime \prime}, x\right)=0$, where $F\left(v, v^{\prime}, v^{\prime \prime}, x\right)$ is a differentiable functional with respect to $v, v^{\prime}$ and $v^{\prime \prime}$. Then, the generalized Newton-Raphson-Kantorovich approximation can be reformulated as

$$
\begin{gathered}
F\left(v_{n+1}, v_{n+1}^{\prime}, v_{n+1}^{\prime \prime}, x\right) \approx F\left(v_{n}, v_{n}^{\prime}, v_{n}^{\prime \prime}, x\right)+\left(v_{n+1}-v_{n}\right) F_{v}\left(v_{n}, v_{n}^{\prime}, v_{n}^{\prime \prime}, x\right) \\
+\left(v_{n+1}^{\prime}-v_{n}^{\prime}\right) F_{v^{\prime}}\left(v_{n}, v_{n}^{\prime}, v_{n}^{\prime \prime}, x\right)+\left(v_{n+1}^{\prime \prime}-v_{n}^{\prime \prime}\right) F_{v^{\prime \prime}}\left(v_{n}, v_{n}^{\prime}, v_{n}^{\prime \prime}, x\right)
\end{gathered}
$$

As a result, the sequence $\left\{v_{n}\right\}$ will converge to the solution of the nonlinear ODE: $F\left(v, v^{\prime}, v^{\prime \prime}, x\right)=0$.

Hence, in order to solve the transformed Falkner-Skan problem (2.6), we consider the functional

$$
\begin{aligned}
F\left(v, v^{\prime}, v^{\prime \prime}, t\right)=(1-t)^{4} v^{\prime \prime} & -2(1-t)^{3} v^{\prime}-\beta_{1} v^{2}+\beta_{1} \\
& +\beta_{0}(1-t)^{2} v^{\prime} \int_{0}^{t} \frac{v(\epsilon)}{(1-\epsilon)^{2}} d \epsilon .
\end{aligned}
$$

Then, the partial derivatives of $F$ are

$$
\begin{aligned}
F_{v}\left(v, v^{\prime}, v^{\prime \prime}, t\right) & =\beta_{0}(1-t)^{2} v^{\prime} \int_{0}^{t} \frac{1}{(1-\epsilon)^{2}} d \epsilon-2 \beta_{1} v, \\
F_{v^{\prime}}\left(v, v^{\prime}, v^{\prime \prime}, t\right) & =-2(1-t)^{3}+\beta_{0}(1-t)^{2} \int_{0}^{t} \frac{1}{(1-\epsilon)^{2}} v(\epsilon) d \epsilon, \\
F_{v^{\prime \prime}}\left(v, v^{\prime}, v^{\prime \prime}, t\right) & =(1-t)^{4} .
\end{aligned}
$$


Thus, the resulting quasi-linearizased problem (2.6) is given by

$$
\begin{aligned}
(1-t)^{4} k_{n}^{\prime \prime} & -2(1-t)^{3} k_{n}^{\prime}+\beta_{0}(1-t)^{2} k_{n}^{\prime} \int_{0}^{t} \frac{1}{(1-\epsilon)^{2}} k_{n}(\epsilon) d \epsilon-\beta_{1} k_{n}^{2}+\beta_{1} \\
& +\left(k_{n+1}-k_{n}\right)\left(\beta_{0}(1-t)^{2} k_{n}^{\prime} \int_{0}^{t} \frac{1}{(1-\epsilon)^{2}} d \epsilon-2 \beta_{1} k_{n}\right) \\
& +\left(k_{n+1}^{\prime}-k_{n}^{\prime}\right)\left(\beta_{0}(1-t)^{2} \int_{0}^{t} \frac{1}{(1-\epsilon)^{2}} k_{n}(\epsilon) d \epsilon-2(1-t)^{3}\right) \\
& +\left(k_{n+1}^{\prime \prime}-k_{n}^{\prime \prime}\right)(1-t)^{4}=0,
\end{aligned}
$$

which can be simplified as

$$
\begin{gathered}
(1-t)^{4} k_{n+1}^{\prime \prime}+\left(\beta_{0}(1-t)^{2} \int_{0}^{t} \frac{1}{(1-\epsilon)^{2}} k_{n}(\epsilon) d \epsilon-2(1-t)^{3}\right) k_{n+1}^{\prime} \\
+\left(\beta_{0} t(1-t) k_{n}^{\prime}-2 \beta_{1} k_{n}\right) k_{n+1}=\beta_{0} t(1-t) k_{n}^{\prime} k_{n}-\beta_{1} k_{n}^{2}-\beta_{1}
\end{gathered}
$$

subject to the boundary conditions

$$
k_{n+1}(0)=\gamma . \quad k_{n+1}(1)=1 .
$$

The main advantage of the proposed iterative scheme is that, given $k_{n}$, the function $k_{n+1}$ is the solution of a linear ordinary differential problem with known Dirichlet boundary conditions. Thus, at every iteration, given $k_{n}$, the obtained linearized ODE will be numerically solved for $k_{n+1}$ using the finite difference method.

Provided that the choice of the initial approximation $k_{0}$ is crucial for the convergence of the scheme, we propose the following initial solution satisfying the boundary conditions $(2.8 \mathrm{~b})$ :

$$
k_{0}(t)=(1-\gamma) t^{2}+\gamma, \quad \text { for } 0 \leq t \leq 1
$$

\subsection{Iterative finite difference scheme}

In order to solve equation (2.8) for $k_{n+1}$ using the finite difference method, we consider a uniform mesh of size $h=\frac{1}{M}$ and grid points $t_{i}=i * h$ for $i=0 \ldots M$. For a fixed $n>0$, we refer to the finite difference solution as $K_{n}$.

To write the finite difference scheme, We use the standard finite difference approximations of the first and second derivatives, respectively, as

$$
k_{n}^{\prime}\left(t_{i}\right) \approx \frac{K_{n}\left(t_{i+1}\right)-K_{n}\left(t_{i-1}\right)}{2 h}, \quad k_{n}^{\prime \prime}\left(t_{i}\right) \approx \frac{K_{n}\left(t_{i+1}\right)-2 K_{n}\left(t_{i}\right)+K_{n}\left(t_{i-1}\right)}{h^{2}},
$$

for $1 \leq i \leq M-1$.

We also use a numerical computation of the integral $\int_{0}^{t} \frac{k_{n}(\epsilon)}{(1-\epsilon)^{2}} d \epsilon$ at the 
mesh points $t_{i}$, for $i=1, \ldots, M-1$, using the standard Simpson's rule as

$$
\begin{aligned}
& \int_{0}^{t_{i}} \frac{k_{n}(\epsilon)}{(1-\epsilon)^{2}} d \epsilon \approx \sum_{j=0}^{i} \frac{K_{n}\left(t_{j}\right)}{\left(1-t_{j}\right)^{2}} W_{j}=I_{n}\left(t_{i}\right), \text { if } i \text { is even } \\
& \int_{0}^{t_{i}} \frac{k_{n}(\epsilon)}{(1-\epsilon)^{2}} d \epsilon \approx \frac{h}{2}\left(\frac{K_{n}\left(t_{1}\right)}{\left(1-t_{1}\right)^{2}}+\gamma\right)+\sum_{j=1}^{i} \frac{K_{n}\left(t_{j}\right)}{\left(1-t_{j}\right)^{2}} W_{j}=I_{n}\left(t_{i}\right), \text { if } i \text { is odd }
\end{aligned}
$$

where $W_{j}$ 's are the known Simpson's weights. Hence, we can write the finite difference scheme for the ODE (2.8) as follows

$$
\begin{aligned}
& \left(\frac{\left(1-t_{i}\right)^{4}}{h^{2}}+\frac{\beta_{0}\left(1-t_{i}\right)^{2} I_{n}\left(t_{i}\right)-2\left(1-t_{i}\right)^{3}}{2 h}\right) K_{n+1}\left(t_{i+1}\right) \\
& \quad+\left(\beta_{0} t_{i}\left(1-t_{i}\right) D K_{n}\left(t_{i}\right)-2 \beta_{1} K_{n}\left(t_{i}\right)-2 \frac{\left(1-t_{i}\right)^{4}}{h^{2}}\right) K_{n+1}\left(t_{i}\right) \\
& \quad+\left(\frac{\left(1-t_{i}\right)^{4}}{h^{2}}-\frac{\beta_{0}\left(1-t_{i}\right)^{2} I_{n}\left(t_{i}\right)-2\left(1-t_{i}\right)^{3}}{2 h}\right) K_{n+1}\left(t_{i-1}\right) \\
& =\beta_{0} t_{i}\left(1-t_{i}\right) D K_{n}\left(t_{i}\right) K_{n}\left(t_{i}\right)-\beta_{1}\left(K_{n}\left(t_{i}\right)\right)^{2}-\beta_{1}, \quad(1 \leq i \leq M-1),
\end{aligned}
$$

where $D K_{n}$ is the centred finite difference approximation of the first derivative using the computed solution from the previous iteration $n$, i.e.,

$$
D K_{n}\left(t_{i}\right) \approx \frac{K_{n}\left(t_{i+1}\right)-K_{n}\left(t_{i-1}\right)}{2 h}, \quad \text { for } 1 \leq i \leq M-1 .
$$

Using the simplified notations $K_{n}^{i}=K_{n}\left(t_{i}\right)$ and $I_{n}^{i}=I_{n}\left(t_{i}\right)$, for $n \geq 0$, we can summarize the iterative finite difference scheme (2.11) above as follows:

$$
\begin{aligned}
& \left(\frac{\left(1-t_{i}\right)^{4}}{h^{2}}+\frac{\beta_{0}\left(1-t_{i}\right)^{2} I_{n}^{i}-2\left(1-t_{i}\right)^{3}}{2 h}\right) K_{n+1}^{i+1} \\
& \quad+\left(\beta_{0} t_{i}\left(1-t_{i}\right) D K_{n}^{i}-2 \beta_{1} K_{n}^{i}-2 \frac{\left(1-t_{i}\right)^{4}}{h^{2}}\right) K_{n+1}^{i} \\
& \quad+\left(\frac{\left(1-t_{i}\right)^{4}}{h^{2}}-\frac{\beta_{0}\left(1-t_{i}\right)^{2} I_{n}^{i}-2\left(1-t_{i}\right)^{3}}{2 h}\right) K_{n+1}^{i-1} \\
& =\beta_{0} t_{i}\left(1-t_{i}\right) D K_{n}^{i} K_{n}^{i}-\beta_{1}\left(K_{n}^{i}\right)^{2}-\beta_{1},
\end{aligned}
$$

for $1 \leq i \leq M-1$. The boundary conditions are written as

$$
K_{n}\left(t_{0}=0\right)=\gamma, \quad K_{n}\left(t_{M}=1\right)=1 .
$$

In addition, the initial approximation is given by

$$
K_{0}\left(t_{i}\right)=(1-\gamma) t_{i}^{2}+\gamma \quad \text { for } 0 \leq i \leq M
$$

Then, once the numerical solution $K_{n}$ of the integro-differential equation (2.6) is computed, the other numerical solution $H_{n}$ of the nonlinear system (2.4) is obtained via a numerical evaluation of (2.5) using (2.9) and (2.10). 
Finally, the actual solutions $f$ and $g$ of Falkner-Skan equation are numerically computed at $\eta_{i}, i=0, \ldots, M$, by using the backward transformation (2.3) as follows

$$
F\left(\eta_{i}\right)=H\left(t_{i}\right), \quad G\left(\eta_{i}\right)=K\left(t_{i}\right), \quad \eta_{i}=\frac{t_{i}}{1-t_{i}} .
$$

We note that, in order to control the convergence of the proposed IFD scheme (2.12a), we set an error tolerance $\delta>0$. Then, the algorithm is iteratively applied until such accuracy is reached in the sense that $\left\|K_{n}-K_{n-1}\right\|_{\infty} \leq \delta$.

We also mention that the convergence rate of the proposed IFD scheme (2.12a) is quadratic, since it is based on Newton-Raphson-Kantorovich approximation [3]. Thus, this scheme is highly efficient in terms of computational time and cost. Such fact will be numerically exhibited in the following Section 3.

\section{Numerical simulation}

In this section, we present several numerical simulations in which we compute the solution of Falkner-Skan equation (1.1) for various values of the parameters $\beta_{0}$ and $\beta_{1}$ corresponding to different cases of flows. Particularly, we focus on the following special cases: Blasius flow [4]: $\beta_{1}=0$; Pohlhausen flow [19]: $\beta_{0}=0$ and $\beta_{1}=1$; Hiemenz flow [12]: $\beta_{0}=1$ and $\beta_{1}=1$; Homann flow [13]: $\beta_{0}=2$ and $\beta_{1}=1$.

In Table 1 , we present the numerical solution of Blasius equation $\left(\beta_{1}=0\right)$ computed with the proposed IFD method when $\beta_{0}=0.5$. We also present a comparison of this computed solution to some of the most accurate results available in the literature $[6,14]$.

Table 1. Numerical solution of Blasius equation with $\beta_{0}=0.5$ and $\beta_{1}=0$ compared to Howarth's [14] and Cortell's [6] solutions.

\begin{tabular}{llllllllll}
\hline$\eta$ & $f(\eta)$ & $f(\eta)[6]$ & $f(\eta)[14]$ & $f^{\prime}(\eta)$ & $f^{\prime}(\eta)[6]$ & $f^{\prime}(\eta)[14]$ & $f^{\prime \prime}(\eta)$ & $f^{\prime \prime}(\eta)[6]$ & $f^{\prime \prime}(\eta)[14]$ \\
\hline 0 & 0.00000 & 0.00000 & 0.00000 & 0.00000 & 0.00000 & 0.00000 & 0.33206 & 0.33206 & 0.33206 \\
1 & 0.16557 & 0.16557 & 0.16557 & 0.32978 & 0.32978 & 0.32979 & 0.32301 & 0.32301 & 0.32301 \\
2 & 0.65003 & 0.65003 & 0.65003 & 0.62977 & 0.62977 & 0.62977 & 0.26675 & 0.26675 & 0.26675 \\
3 & 1.39681 & 1.39682 & 1.39682 & 0.84605 & 0.84605 & 0.84605 & 0.16136 & 0.16136 & 0.16136 \\
4 & 2.30575 & 2.30576 & 2.30576 & 0.95552 & 0.95552 & 0.95552 & 0.06423 & 0.06423 & 0.06424 \\
5 & 3.28333 & 3.28330 & 3.28329 & 0.99154 & 0.99155 & 0.99155 & 0.01591 & 0.01591 & 0.01591 \\
6 & 4.27967 & 4.27965 & 4.27964 & 0.99897 & 0.99898 & 0.99898 & 0.00240 & 0.00240 & 0.00240 \\
7 & 5.27925 & 5.27927 & 5.27926 & 0.99992 & 0.99993 & 0.99992 & 0.00022 & 0.00022 & 0.00022 \\
8 & 6.27929 & 6.27925 & 6.27923 & 0.99999 & 1.00000 & 1.00000 & 0.00001 & 0.00001 & 0.00001 \\
9 & 7.27922 & 7.27925 & 7.27923 & 1.00000 & 1.00000 & 1.00000 & 0.00000 & 0.00000 & 0.00000 \\
\hline
\end{tabular}

In Table 2 we present the numerical solution of Blasius equation $\left(\beta_{1}=0\right)$ computed with the proposed IFD method for the values of $\beta_{0}=2 / 3$ and $\beta_{0}=1$. We compare it to the results in [6]. It is clear that our computed solution is accurate and in good agreement with the previously computed solutions of Falkner-Skan problem.

In Table 3, we present the computed values of the wall shear stress $\alpha=f^{\prime \prime}(0)$ for Falkner-Skan model when $\beta_{0}=1$, for different values of $\beta_{1}$. We also compare 
Table 2. Numerical solution of Blasius equation with $\beta_{1}=0$ compared to Cortell's [6] solution.

\begin{tabular}{clllllll}
\hline$\beta_{0}$ & $\eta$ & $f(\eta)$ & $f(\eta)[6]$ & $f^{\prime}(\eta)$ & $f^{\prime}(\eta)[6]$ & $f^{\prime \prime}(\eta)$ & $f^{\prime \prime}(\eta)[6]$ \\
\hline \multirow{6}{*}{1} & 0 & 0.00000 & 0.00000 & 0.00000 & 0.00000 & 0.46960 & 0.46960 \\
& 1 & 0.23299 & 0.23299 & 0.46063 & 0.46063 & 0.43438 & 0.43438 \\
& 2 & 0.88680 & 0.88681 & 0.81669 & 0.81670 & 0.25567 & 0.25567 \\
& 3 & 1.79557 & 1.79558 & 0.96906 & 0.96906 & 0.06771 & 0.06771 \\
& 4 & 2.78389 & 2.78390 & 0.99777 & 0.99777 & 0.00687 & 0.00687 \\
& 5 & 3.78328 & 3.78325 & 0.99994 & 0.99994 & 0.00026 & 0.00026 \\
& 6 & 4.78326 & 4.78324 & 1.00000 & 1.00000 & 0.00000 & 0.00000 \\
\hline \multirow{4}{*}{} & 0 & 0.00000 & 0.00000 & 0.00000 & 0.00000 & 0.38342 & 0.38342 \\
& 1 & 0.19090 & 0.19090 & 0.37939 & 0.37939 & 0.36747 & 0.36747 \\
& 2 & 0.74235 & 0.74235 & 0.70789 & 0.70789 & 0.27420 & 0.27420 \\
2 & 3 & 1.56317 & 1.56317 & 0.90911 & 0.90910 & 0.12858 & 0.12858 \\
& 4 & 2.51730 & 2.51730 & 0.98323 & 0.98323 & 0.03313 & 0.03313 \\
& 5 & 3.51042 & 3.51038 & 0.99827 & 0.99827 & 0.00444 & 0.00444 \\
& 6 & 4.50983 & 4.50979 & 0.99990 & 0.99990 & 0.00030 & 0.00030 \\
& 7 & 5.50976 & 5.50976 & 0.99999 & 0.99999 & 0.00001 & 0.00001 \\
& 8 & 6.50983 & 6.50977 & 1.00000 & 1.00000 & 0.00000 & 0.00000 \\
\hline
\end{tabular}

these results obtained by our IFD method to some existing accurate results available in the literature $[1,26]$.

Table 3. The wall shear stress $\alpha=f^{\prime \prime}(0)$ of Falkner-skan model with $\beta_{0}=1$ compared to Asaithambi's [1] and Salama's [26] solutions.

\begin{tabular}{llll}
\hline$\beta_{1}$ & $\alpha$ & $\alpha[1]$ & $\alpha[26]$ \\
\hline 40 & 7.314802 & 7.314785 & 7.314785 \\
30 & 6.338220 & 6.338209 & 6.338208 \\
20 & 5.180725 & 5.180718 & 5.180718 \\
15 & 4.491492 & 4.491487 & 4.491487 \\
10 & 3.675237 & 3.675234 & 3.675234 \\
2 & 1.687218 & 1.687218 & 1.687218 \\
1 & 1.232587 & 1.232589 & 1.232588 \\
0.5 & 0.927679 & 0.927680 & 0.927680 \\
0 & 0.469599 & 0.469600 & 0.469600 \\
\hline
\end{tabular}

In Table 4 we consider different benchmark solutions for some of the famous practical cases of flows.

Furthermore, in Figure 1, we graphically exhibit the solution of FalknerSkan problem for different flows (Blasius, Pohlhausen, Hiemenz, and Homann). It is observed that for all these types of flows, the solution of Falkner-Skan problem is increasing with respect to $\eta$.

We also display the velocity profile in Figure 2 as well as the shear stress in Figure 3 for different flows. We notice that for all the considered types of flows, the shear stress decays to zero, however the decay in the case of Blasius flow is slower than in the other flow cases. 
Table 4. The wall shear stress $\alpha=f^{\prime \prime}(0)$ of Falkner-skan models compared to different benchmark solutions.

\begin{tabular}{lccll}
\hline Type of flow & $\beta_{0}$ & $\beta_{1}$ & & $\alpha$ \\
\hline Blasius flow & $\frac{1}{2}$ & 0 & 0.33206 & $0.33206[6]$ \\
Pohlhausen flow & 0 & 1 & 1.15470 & $1.15470[19]$ \\
Hiemenz flow & 1 & 1 & 1.23258 & $1.23258[29]$ \\
Homann flow & 2 & 1 & 1.31193 & $1.31193[13]$ \\
\hline
\end{tabular}

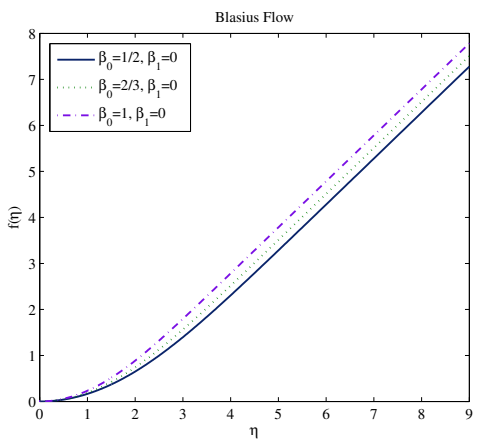

(a) $\mathrm{t}=1$

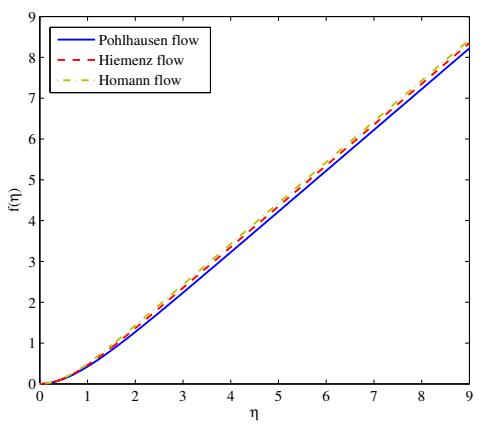

(b) $\mathrm{t}=1$

Figure 1. The Solution of Falkner-Skan problem for the cases of: Blasius problem $\beta_{1}=0$ (left) and Pohlhausen, Hiemenz, and Homann flows $\beta_{0}=0,1,2$, respectively, with $\beta_{1}=1$ (right).

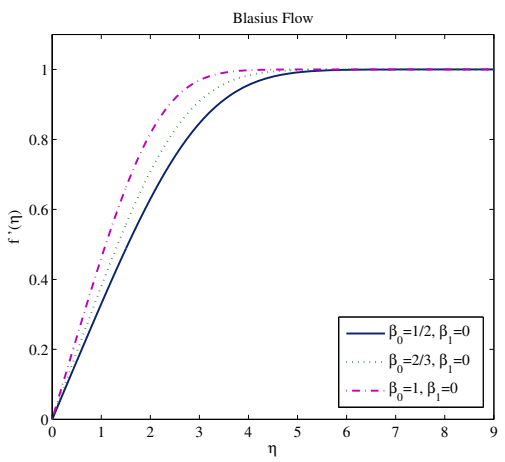

(a) $\mathrm{t}=1$

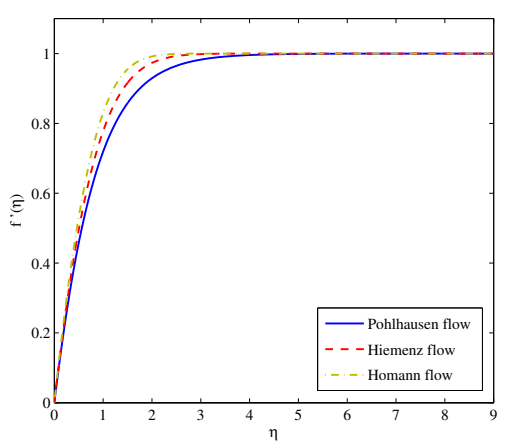

(b) $\mathrm{t}=8$

Figure 2. Velocity profile of Falkner-Skan solution for the cases of: Blasius problem $\beta_{1}=0$ (left) and Pohlhausen, Hiemenz, and Homann flows $\beta_{0}=0,1,2$, respectively, with $\beta_{1}=1$ (right). 


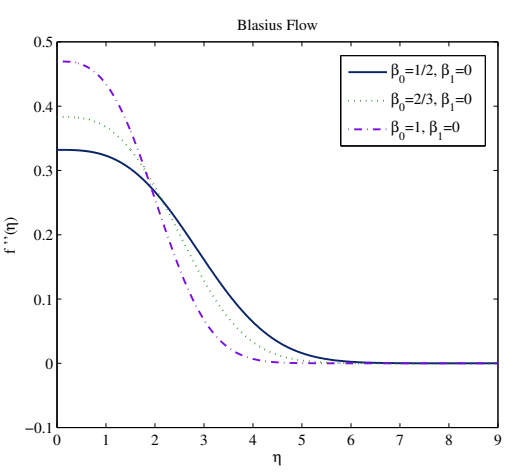

(a) $\mathrm{t}=1$

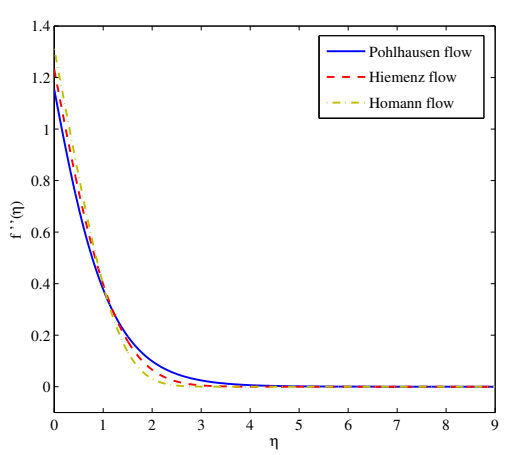

(b) $\mathrm{t}=8$

Figure 3. Shear stress of Falkner-Skan solution for the cases of: Blasius problem $\beta_{1}=0$ (left) and Pohlhausen, Hiemenz, and Homann flows $\beta_{0}=0,1,2$, respectively, with $\beta_{1}=1$ (right).

\section{Conclusions}

In this paper, we have presented a new iterative finite difference method to numerically solve a nonlinear problem defined on a semi-infinite domain, arising from the boundary layer flow with stream-wise pressure gradient, known as Falkner-Skan problem.

For that purpose, we have introduced a new coordinate transformation to overcome the difficulty of the semi-infinite domain. Such transformation led to a second order nonlinear integro-differential equation defined on a finite domain and subjected to boundary conditions on finite values.

We have then used Newton-Raphson-Kantorovich quasilinearisation method in function space [3] in combination with the standard finite difference method to solve transformed Falkner-Skan problem. The proposed iterative finite difference method leads to an iterative scheme in which an accurate solution at every iteration is computed using the approximate solution from the previous iteration.

\section{References}

[1] A. Asaithambi. Solution of the Falkner-Skan equation by recursive evaluation of Taylor coefficients. Journal of Computational and Applied Mathematics, 176(1):203-214, 2005. https://doi.org/10.1016/j.cam.2004.07.013.

[2] N.S. Asaithambi. A numerical method for the solution of the FalknerSkan equation. Applied Mathematics and Computation, 81(2):259-264, 1997. https://doi.org/10.1016/S0096-3003(95)00325-8.

[3] R.E. Bellman and R.E. Kalaba. Quasilinearization and Nonlinear BoundaryValue Problems. American Elsevier Publishing Company, New York, 1965.

[4] H. Blasius. Grenzschichten in Flüssigkeiten mit kleiner Reibung. Zeitschrift für angewandte Mathematik und Physik, 56:1-37, 1908. 
[5] T. Cebeci and H. Keller. Shooting and parallel shooting methods for solving the Falkner-Skan boundary-layer equations. Journal of Computational Physics, 7(2):289-300, 1971. https://doi.org/10.1016/0021-9991(71)90090-8.

[6] R. Cortell. Numerical solutions of the classical Blasius flat-plate problem. Applied Mathematics and Computation, 170(1):706-710, 2005. https://doi.org/10.1016/j.amc.2004.12.037.

[7] V.M. Falkner and S.W. Skan. LXXXV. solutions of the boundary-layer equations. The London, Edinburgh, and Dublin Philosophical Magazine and Journal of Science, 12(80):865-896, 1931. https://doi.org/10.1080/14786443109461870.

[8] T. Fang, W. Liang and C.F. Lee. A new solution branch for the Blasius equation - A shrinking sheet problem. Computers $\&$ Mathematics with Applications, 56(12):3088-3095, 2008. https://doi.org/10.1016/j.camwa.2008.07.027.

[9] T. Fang and J. Zhang. An exact analytical solution of the FalknerSkan equation with mass transfer and wall stretching. International Journal of Non-Linear Mechanics, 43(9):1000-1006, 2008. https://doi.org/10.1016/j.ijnonlinmec.2008.05.006.

[10] R. Fazio. The iterative transformation method for the Sakiadis problem. Computers \& Fluids, 106(SupplementC):196-200, 2015. https://doi.org/10.1016/j.compfluid.2014.10.007.

[11] D. Hartree. On an equation occurring in Falkner and Skan's approximate treatment of the equations of the boundary layer. Mathematical Proceedings of the Cambridge Philosophical Society, 33(2):223-239, 1937. https://doi.org/10.1017/S0305004100019575.

[12] K. Hiemenz. Die Grenzschicht an einem in den gleichförmigen Flüssigkeitsstrom eingetauchten geraden Kreiszylinder. Polytechnisches Journal, 326:391-393, 1911.

[13] F. Homann. Der Einfluss grosser Zähigkeit bei der Strömung um den Zylinder und um die Kugel. Zeitschrift für Angewandte Mathematik und Mechanik, 16(3):153-164, 1936. https://doi.org/10.1002/zamm.19360160304.

[14] L. Howarth. On the solution of the laminar boundary layer equations. Proceedings of the Royal Society of London A : Mathematical, Physical and Engineering Sciences, 164(919):547-579, 1938. https://doi.org/10.1098/rspa.1938.0037.

[15] S. Liao. A uniformly valid analytic solution of two-dimensional viscous flow over a semi-infinite flat plate. Journal of Fluid Mechanics, 385:101-128, 1999. https://doi.org/10.1017/S0022112099004292.

[16] S. Liao. Beyond Perturbation. Introduction to the Homotopy Analysis Method. Chapman Hall/CRC, Boca Raton, 2003. https://doi.org/10.1201/9780203491164.

[17] C.-S. Liu and J.-R Chang. The Lie-group shooting method for multiple-solutions of Falkner-Skan equation under suction-injection conditions. International Journal of Non-Linear Mechanics, 43(9):844-851, 2008. https://doi.org/10.1016/j.ijnonlinmec.2008.05.005.

[18] S. Mukhopadhyay, I.C. Mondal and A.J. Chamkha. Casson fluid flow and heat transfer past a symmetric wedge. Heat Transfer-Asian Research, 42(8):665-675, 2013. https://doi.org/10.1002/htj.21065.

[19] K. Pohlhausen. Zur näherungsweisen Integration der Differentialgleichung der laminaren Grenzschicht. Journal of Applied Mathematics and Mechanics (ZAMM), 1(4):252-268, 1921. https://doi.org/10.1002/zamm.19210010402. 
[20] C.S.K. Raju, M.M. Hoque and T. Sivasankar. Radiative flow of Casson fluid over a moving wedge filled with gyrotactic microorganisms. Advanced Powder Technology, 28(2):575-583, 2017. https://doi.org/10.1016/j.apt.2016.10.026.

[21] C.S.K. Raju, S.M. Ibrahim, S. Anuradha and P. Priyadharshini. Bio-convection on the nonlinear radiative flow of a Carreau fluid over a moving wedge with suction or injection. The European Physical Journal Plus, 131(11):409-409, 2016. https://doi.org/10.1140/epjp/i2016-16409-7.

[22] C.S.K. Raju and N. Sandeep. A comparative study on heat and mass transfer of the Blasius and Falkner-Skan flow of a bio-convective Casson fluid past a wedge. The European Physical Journal Plus, 131(11):405, 2016. https://doi.org/10.1140/epjp/i2016-16405-y.

[23] C.S.K. Raju and N. Sandeep. Nonlinear radiative magnetohydrodynamic Falkner-Skan flow of Casson fluid over a wedge. Alexandria Engineering Journal, 55(3):2045-2054, 2016. https://doi.org/10.1016/j.aej.2016.07.006.

[24] C.S.K. Raju and N. Sandeep. MHD slip flow of a dissipative Casson fluid over a moving geometry with heat source/sink: A numerical study. Acta Astronautica, 133:436-443, 2017. https://doi.org/10.1016/j.actaastro.2016.11.004.

[25] P.L. Sachdev, R.B. Kudenatti and N.M. Bujurke. Exact analytic solution of a boundary value problem for the Falkner-Skan equation. Studies in Applied Mathematics, 120(1):1-16, 2008. https://doi.org/10.1111/j.1467-9590.2007.00386.x.

[26] A.A. Salama. Higher-order method for solving free boundary-value problems. Numerical Heat Transfer, Part B: Fundamentals, 45(4):385-394, 2004. https://doi.org/10.1080/10407790490278002.

[27] I. Sher and A. Yakhot. New approach to solution of the Falkner-Skan equation. American Institute of Aeronautics and Astronautics, 39(1):965-967, 2001. https://doi.org/10.2514/2.1403.

[28] H. Temimi and M. Ben-Romdhane. An iterative finite difference method for solving Bratu's problem. Journal of Computational and Applied Mathematics, 292 (SupplementC):76-82, 2016. https://doi.org/10.1016/j.cam.2015.06.023.

[29] K.A. Yih. The effect of uniform suction/blowing on heat transfer of magnetohydrodynamic hiemenz flow through porous media. Acta Mechanica, 130(3):147158, 1998. https://doi.org/10.1007/BF01184307.

[30] S. Zhu, Q. Wu and X. Cheng. Numerical solution of the Falkner-Skan equation based on quasi-linearization. Applied Mathematics and Computation, 215(7):2472-2485, 2009. https://doi.org/10.1016/j.amc.2009.08.047.

[31] S. Zhu, H. Zhu, Q. Wu and Y. Khan. An adaptive algorithm for the Thomas-Fermi equation. Numerical Algorithms, 59(3):359-372, 2012. https://doi.org/10.1007/s11075-011-9494-1. 